\title{
Lighting and Light Sources
}

AMNG the papers presented in Section 6 of the International Electrical Congress, recently held in Paris, was one by Dr. Pirani, of the Osram Gesellschaft, who gave a very interesting account of recent developments in the production of light. After a general description of the underlying principles of the modern discharge tube, he dealt in more detail with the two types showing the greatest efficiency, namely, the sodium tube and the mercury tube. In the case of the former it has been found experimentally that the addition of one of the rare gases at a pressure about a thousand times as great as that of the sodium vapour is of considerable advantage. Since the atoms of the rare gas require a very high voltage to excite them, they act principally as elastic obstacles in the path of the electrons and so increase a hundred-fold the distance which an electron travels on its journey from the cathode to the anode. The chance of a collision between the electron and an atom of sodium is thus correspondingly increased. When the tube is operating under the most favourable conditions, 70-80 per cent of the energy of the electrons is transformed into light. An over-all efficiency of about sixty lumens a watt can be obtained from a sodium tube.

In the case of the mercury tube, the intensities of the lines in the longer wave-length part of the spectrum are much increased, relatively to the others, by increasing the pressure in the tube to one atmosphere or more. This is illustrated by diagrams showing the relative intensities of the different lines for two tubes operating at pressures of $0.5 \mathrm{~mm}$. and 3 atmospheres respectively. The result is naturally a very great increase in luminous efficiency and lamps can be operated to give 50 lumens a watt. The possibilities of light production by the excitation of gases in the molecular state, and of liquids or solids, are discussed by the author in a final section of his paper, but he concludes that the most hopeful line of work lies with gases in the atomic state or with vapours.

A new type of mercury arc in quartz, stated to have an efficiency of some 10 candles a watt, was described in a paper by Prof. Cz. Reczynski of Poland. It depends on the reduction of the cathode potential drop from 100 volts to about 5 volts by $(a)$ using for the cathode a ball of tungsten instead of mercury, and $(b)$ placing a tungsten filament at a short distance away from the cathode.

In an interesting communication from Japan, Dr. R. Kurosawa described a method of studying the characteristics of a diffusing material, as regards transmission and reflection. The brightness (not the candle-power) of a sample of the material is measured at all angles of view, the light being incident normally.
The measurements are most conveniently carried out by means of a photoelectric cell (that employed by the author is a vacuum type cæsium cell), an image of a given area of the surface of the material being formed on the sensitive surface of the cell. The author recommends Halbertsma's definition of diffusing power $(\sigma)$, that is,

$$
\sigma=\Sigma^{n} \beta(\theta) / n \beta(o)
$$

where $\beta(\theta)$ is the brightness at an angle $\theta$ to the normal and $n$ is the number of directions in which measurements are made. The author considers that three measurements, at the angles $22 \frac{1}{2}^{\circ}, 45^{\circ}$, and $671^{\circ}$, are generally sufficient. Since $\beta(0)$ cannot be obtained by direct measurement in the case of reflection, it must be deduced by extrapolation from observations made at the above three angles, using Lagrange's formula $\beta(0)=\sum^{3} R_{i} \beta\left(\frac{i \pi}{8}\right)$ where $R_{1}=1.5412, R_{2}=$ $-0.6682, R_{3}=0.1270$. A nomogram is given in the paper for finding $\sigma$ from the observed values of $\beta_{2} / \beta_{1}$ and $\beta_{3} / \beta_{1}$, using the formula deduced by the above method, namely,

$$
\sigma=\left(\beta_{1}+\beta_{2}+\beta_{3}\right) /\left(4 \cdot 62 \beta_{1}-2 \cdot 00 \beta_{2}+0 \cdot 38 \beta_{3}\right) .
$$

The subject of glare in lighting installations was considered in two papers presented in this section. M. J. Dourgnon, defining glare as the threshold sensitivity of the retina at a given point $A$ (for example, the fovea) with the eye exposed to any given field, proposed that the glare produced by a uniform field having a brightness equal to 1 candle per square $\mathrm{cm}$. should be adopted as the 'unit of glare'.

Ing. J. Ondracek, of Austria, dealt principally with the time required for the eye to adapt itself when the gaze was transferred from a surface of high brightness to one of low brightness, or vice versa. This time is a function of the average brightness of the two fields of view and may be expressed in terms of the ratio of these two brightnesses. In the practical case, one brightness is that of the surface being worked upon, while the other is the average brightness of the surroundings, for example, ceiling, walls, and floor.

The use of photoelectric cells for colorimetry was described in a paper by Dr. N. R. Campbell, who outlined the method of colour-matching electric lamps by the use of two cells having markedly different sensitivity curves, and the use of the Toussaint photoelectric colorimeter for giving a rough indication of the spectral distribution of the light reflected from a coloured surface.

\section{Evaporation, Condensation, and Adsorption}

DR. I. LANGMUIR ( $J$. Amer. Chem. Soc., July) has extended his well-known adsorption formula by taking account of the forces between adjacent adsorbed particles ('adatoms'). In the simple theory, the rate of evaporation of adatoms from a surface is $\nu_{1} \theta$, where $\theta$ is the fraction of the surface covered and $v_{1}$ a constant. If atoms condense only on uncovered. parts, the rate of condensation is $\alpha_{0} \mu(1-\theta)$, where $\alpha_{0}$ is constant, measuring the efficiency of condensation on a bare surface, and $\mu$ the rate of collision of incident atoms per unit area of surface, given by the kinetic theory :

$$
\mu=(2 \pi m k T)^{-\frac{1}{2}} p=2.653 \times 10^{19} p(M T)^{-\frac{1}{2}} .
$$

where $p=$ pressure in baryes, $k=$ Boltzmann's conNo. 3283, VoL. 130] stant, $m$ is the mass of an atom, and $M$ the atomic weight of the gas. For steady states the rates of evaporation and condensation are equal, hence

$$
\theta=\alpha_{0} \mu /\left(\nu_{1}+\alpha_{0} \mu\right)=\alpha_{0} \tau \mu /\left(\sigma_{1}+\alpha_{0} \tau \mu\right)
$$

where $\tau=\sigma_{1} / \nu_{1}=$ average life of an adatom, $\sigma_{1}$ being the number of adatoms per unit area of saturated surface $(\theta=1)$. Equation (2) represents the observed adsorption on plane surfaces with reasonable accuracy in a surprisingly large number of cases.

The vapour pressure of a liquid is given over a wide range of temperature by

$$
p=A T^{\gamma} e^{-b i T} \simeq A_{\theta} e^{-b_{0} / T} .
$$

Trouton's rule requires that $\gamma=0$ and $A$ is a universal 
constant; Hildebrand's rule that $\gamma=1$ and $A$ a universal constant. A comparison with experimental data for liquids shows that Hildebrand's rule gives better results than Trouton's, but a rule which gives still better agreement is obtained by putting $\gamma=1.5$, when $\log A_{1 \cdot 5}=6 \cdot 37$. The vapour pressures of solids, the vapours of which have rigid molecules, are also given by this equation with $\gamma=1.5$ and $\log A=6.9$, but much larger values of $A$ are obtained if the molecules possess internal degrees of freedom. It is therefore assumed that such molecules in the vapour phase may possess high internal mobility, as though liquid, whilst at lower temperatures they may become rigid, as though solid. Such effects probably do not exist with molecules of vapours of liquids. This part of the paper contains a detailed and valuable analysis of experimental data.

The ratio of the latent heat of fusion to the melting point has high values for large molecules such as stearic acid, increasing roughly in proportion to the number of atoms in the molecule, and in such cases a large part of the heat of fusion represents an internal heat of fusion of the molecules themselves, which in the solid are rigidly arranged within the lattice, so that the molecule itself is solid, but when the solid melts the molecule also melts.

In considering the evaporation of adsorbed atoms from an adsorbed film containing $\sigma$ atoms per unit area, the value of $\tau$, the average life of the adatom, being the same for all, the rate of evaporation (atoms $\mathrm{cm}^{-2}$ sec. $^{-1}$ ) is

$$
\nu=\sigma / \tau=\sigma_{1} \theta / \tau
$$

From (3) in the form

$$
\begin{gathered}
p=A_{1 \cdot 5} T e^{3} e^{-b / T} \\
\text { where } \quad b=b_{0}-\frac{3}{2} T=\frac{\lambda}{k}-\frac{3}{2} T,
\end{gathered}
$$

$\lambda$ being the latent heat of evaporation per atom, and (1) in which $\mu=\nu_{1}=\sigma_{1} / \tau$, we find

$$
\tau=(2 \pi m k)^{\frac{1}{2}}\left(A_{1 \cdot 5} T\right)^{-1} \sigma_{1} e^{-b / T},
$$

in which it is shown that $A_{1 \cdot 5} \simeq 8 \times 10^{6}$, and hence

$$
\nu=A_{1 \cdot 5}(2 \pi m k)^{-\frac{1}{2} \theta} \theta T^{\prime} e^{-b^{\prime} T},
$$

an equation for the rate of evaporation of atoms or molecules from monatomic films on surfaces which is shown to agree reasonably well with experiment for thorium, oxygen, and cæsium films on tungsten. In (4) the forces of interaction between adatoms are taken into account by the value of $b$, and since $b$ is in general a function of $\theta$, the value of $\nu$ is not proportional to $\theta$ except at such low values of $\theta$ that $b$ is near the limiting value for $\theta=0$.

Although the conditions in which adsorbed films more than one molecule thick can be formed are rather unusual, they are discussed. In general, adsorbed molecules on plane homogeneous solids are acted upon by strong forces originating from the underlying solid. The adsorbed molecules thus become polarised and repel one another as dipoles with forces proportional to $M^{2} r^{-4}(M=$ dipole moment; $\boldsymbol{r}=$ distance), and attractive forces predominate only when two kinds of adsorbed molecules are present which become polarised in opposite senses, as cæsium and oxygen on tungsten or salts on metals, such as mercurous sulphate on mercury. In some cases, however, the forces exerted between solid and adatom are small, as when hydrogen molecules or helium atoms strike a chemically saturated surface such as tungsten covered with adsorbed oxygen. In such cases the average life of the adatom is so small that it does not even reach thermal equilibrium with the solid, so that the accommodation coefficient is much less than unity $(0 \cdot 1-0 \cdot 2)$.

The equation of state of the two-dimensional gas composing the adsorbed film may be found by. the virial method with a repulsive force specified in terms of dipoles :

$$
F A=R T+\frac{1}{2} \Sigma(r f) \text {. }
$$

A two-dimensional van der Waals equation, in which the long-range forces are now repulsive, takes the form

$$
\left(F-a / A^{2}\right)\left(A-A_{1}\right)=R T,
$$

in which $A$ is the area containing $1 \mathrm{gm}$. atom and $A_{1}$ in the ordinary derivation considering only first order effects is found to be only half the area actually covered by $1 \mathrm{gm}$. atom of adatoms. Experimental data for oil films at high surface concentrations show that $A_{1}$ corresponds with a close packed film in which the molecules cover the surface completely, and a new theoretical derivation for the case of a high concentration of adatoms confirms this.

The choice of a dipole repulsive force for the virial expression is justified by the experimental result that adsorption of alkali metal atoms occurs strongly only when the electron affinity of the adsorbent metal exceeds the ionising potential of the alkali metal. The positive charge on the adatoms causes a change in contact potential by as much as 3 volts and a corresponding increase in electron emission. It is then shown that it is possible to calculate the moments $M$ as functions of $\theta$. In the case of cæsium on tungsten, these vary from 16 debyes for $\theta=0$ to 6 debyes for $\theta=0.9$. The electron and positive ion emission rates are then calculated and found to be in agreement with experiment. In the first case, the influence of electron spin is taken into account and Dushman's equation, derived from the Sackur-Tetrode relation, is somewhat modified, although probably within the limits of experimental error.

The results on the evaporation of cæsium films are very different from those predicted by the old formula (2), based on the assumption that there are no repulsions between adatoms, but the general results are in agreement with the new equations.

The effect of inhomogeneity of the adsorbing surface, first predicted by Langmuir and since studied experimentally and theoretically by H. S. Taylor and others, is then considered. The importance of the socalled 'active areas' in determining the catalytic properties of surfaces, even plane surfaces, is well known. It is shown, however, that the calculations lead to the result that the tungsten surface is essentially homogeneous, although they indicate that on about $\mathbf{0 . 5}$ per cent of the surface the cæsium atoms are much more firmly bound than the rest. The active spots probably consist of isolated elementary surfaces each capable of holding one adatom.

\section{University and Educational Intelligence}

CAmbridge.-The Busk studentship in aeronautics, founded in memory of Edward Teshmaker Busk, who lost his life in 1914 whilst flying an experimental aeroplane, has been awarded for the year 1932-33 to Mr. Herbert Brian Squire, of Balliol College, Oxford.

London.-The Coal and Corn and Finance Committee of the Common Council of the Corporation of the City of London has recommended the grant of $£ 100,000$ towards the new central buildings of the University which are to be erected in Bloomsbury (see Nature of July 9, p. 49). At Lord Macmillan's suggestion, the gift of the Corporation will be devoted

No. 3283, Vou. 130] 